\title{
Early Intervention in the Neurodevelopment of Premature Infants during the First Six Months of Life
}

\author{
Irma Alvarado-Guerrero ${ }^{1}$, Adrián Poblano ${ }^{2,3}$, Erzsebet Marosi ${ }^{4}$, María Corsi-Cabrera ${ }^{5}$, \\ Gloria A. Otero-Ojeda ${ }^{6}$
}

\begin{abstract}
${ }^{1}$ Interdisciplinary Center for Early Intervention, College of High Studies at Iztacala (UNAM), Tlalnepantla, Mexico; ${ }^{2}$ Clinic of Sleep Disorders, National University of Mexico (UNAM), Mexico City, Mexico; ${ }^{3}$ Laboratory of Cognitive Neurophysiology, National Institute of Rehabilitation (INR), Mexico City, Mexico; ${ }^{4}$ Department of Neuroscience, College of High Studies at Iztacala (UNAM), Tlalnepantla, Mexico; ${ }^{5}$ School of Psychology, National University of Mexico (UNAM), Mexico City, Mexico; ${ }^{6}$ School of Medicine, University of State of Mexico (UAEM), Toluca, Mexico.

Email: drdeaf@latinmail.com
\end{abstract}

Received October 27 $7^{\text {th }}, 2010$; revised March 16 ${ }^{\text {th }}, 2011$; accepted March 30 ${ }^{\text {th }}, 2011$.

\begin{abstract}
Objective: measure the effects of Early Intervention ad modum Katona (EI-K) in high-risk premature infants by means of clinical, neurobehavioral, and neurophysiologic tests. Method: we used the Amiel-Tison neurologic examination, the Bayley Scale of Infant Behavior, and electroencephalography (EEG) recordings at 42 weeks of conceptional age, and after 6 months of treatment EI-K $(n=14)$ and compared these results with those of a group of infants without early intervention $(n E I)(n=11)$. Results: we found better performance of infants in EI-K than $n E I$ group after 6 months of treatment in neurologic and behavioral examination measurements, but found no differences in EEG comparisons. Conclusion: our data suggest significant benefit of the use of EI-K program over n-EI in the neurologic and neurobehavior examinations of premature infants after 6 months of age.
\end{abstract}

Keywords: Premature, High-risk Infants, Early Intervention, Brain Plasticity

\section{Introduction}

Survival of premature infants, especially low-birth-weight infants has resulted in an increased incidence of neurologic sequelae including cerebral palsy, epilepsy, deafness, blindness, mental retardation, language, and behavioral, and learning disorder [1]. Thus, early detection of children at high-risk is of paramount importance for planning intervention and habilitation studies in an attempt to avoid secondary problems for these individual $[2,3]$.

Early intervention (EI) consists of multidisciplinary services provided to infants from birth through the first years of life to promote child health, enhance emerging competencies, minimize developmental delays, cure existing disabilities, prevent functional deterioration, and promote adaptive parenting functioning. These goals are accomplished by individualized developmental, educational, and therapeutic services. An increased number of investigations have studied the effects of EI on the be- havior and development of high-risk infants (mainly premature infants with low-birth-weight) based on the rationale that premature infants are at risk for neurologic and psychologic disabilities and that customary care may not include adequate environmental stimulation [4]. However, results from several investigations are not conclusive, and many investigators recommend the study of specific developmental training techniques to find evidence of positive effects of EI on neurodevelopment.

Neuromotor-status assessment of high-risk infants during the neonatal period has been found to correlate well with clinical outcome during developmental follow-up [5-7]. Moreover, neurophysiologic tests, such as the electroencephalogram (EEG), provide a quantitative and extremely useful non-invasive prove for brain function. Degree of background- activity abnormality has proved to be a predictor of long-term neurologic outcome $[8,9]$. Thus, we decided to employ these techniques to study the effects of EI in neurodevelopment of a group of premature infants followed during their first year of life. 
The goals of this paper comprise measuring the effects of the EI ad modum Katona (EI-K) program in a group of high-risk premature infants $[10,11]$ by means of clinic and neurophysiologic tests. Our working hypothesis was that high-risk infants under EI-K perform better than a group of high-risk infants without EI treatment (nEI). Clinical, behavioral and EEG tests were used prospectively to evaluate the effects of EI on their neurodevelopment during follow-up in the first year of life.

\section{Method}

\subsection{Subjects}

Newborns from the Hospital of Gynecology and Obstetrics No. 3, at the Institute of the Mexican Social Security (IMSS) in Mexico City were recruited to study the effects of EI-K program. The sample was obtained by convenience. Inclusion criteria were the following: 1) age at birth between 34 and -36 gestational weeks [12]; 2) adequate weight for gestational age at birth [13]; 3) single delivery; and 4) normal result in cranial ultrasonographic (US) scan [14]. Infants were rejected from study if they had the following antecedents: 1) maternal history of high blood pressure, diabetes or another chronic maternal disease during pregnancy; 2) genetic alterations; or 3) intrauterine or neonatal infections; 4) evident external malformations. Clinical characteristics of infants are shown in Table 1. Infants in this report included babies born consecutively over 1 year of work. Other clinical data were obtained from hospital records; all evaluations of these infants were performed correcting age to conceptional age (age at birth + age of life in weeks). Because is not ethical leave an infant without treatment, the comparison group was composed of premature infants with identical inclusion and exclusion criteria whose parents rejected the infant's participation during the first months of life but who accepted EI participation of their child later and examinations at 6 months of age. The group that does not received EI after evaluation at birth, parents were given with recommendations on EI to promote development in: emotional, gross and fine motor behavior, and language areas. Moreover, they received free of charge, a book of EI, and assisted to conferences of infant normal development in University campus. Inclusion of the infant in each group was determined by the parental choice. Eleven infants constituted the n-EI group (six male and five female), while 14 infants (eight males and six females) comprised the EI-K group, differences in the size of the groups are attributable to the parent election to receive EI in the first 6 months of life. The Institutional Review Board approved the study protocol, and parents of infants signed informed consent.
Table 1. Clinical features of infants participating in the early intervention ad modum Katona (EI-K) program and of infants without early intervention (nEI) program during the first 6 months of life.

\begin{tabular}{ccc}
\hline Variable & $\begin{array}{c}\text { EI-K group } \\
x \pm(\mathrm{SD})\end{array}$ & $\begin{array}{c}\text { nEI group } \\
x \pm(\mathrm{SD})\end{array}$ \\
\hline Age at birth (weeks) & $34.9(3.97)$ & $34.6(3.82)$ \\
Weight $(\mathrm{g})$ & $2,227(723)$ & $2,222(813)$ \\
Cephalic perimeter $(\mathrm{cm})$ & 32.3 & $32.1(3.76)$ \\
1 - min Apgar score & $7.3(2.29)$ & $7.5(2.52)$ \\
5 - min Apgar score & $8.5(1.41)$ & $8.6(1.64)$ \\
\hline
\end{tabular}

$x=$ mean $; \mathrm{SD}=$ Standard deviation.

\subsection{Early Intervention}

Patients received K-EI neurohabililtation according to their symptoms early after hospital discharge ad modum Katona. The individually adjusted program was described to the parents (especially to the mother), who were trained and received written programs elaborated for their infants. These programs contain intensive schedules to develop elementary sensori-motor patterns $[15,16]$ and exercises in two main areas: verticalization, and locomotion, and extending to vision, hearing, feeding, and vocalization according to patient needs, at least 6 hours throughout the day, according to the infant's feeding and sleep- time schedules. It was emphasized that, aside from the training programs, the infant requires the affection and care of the family members. Moreover, the training facilitated the mother-infant relationship $[10,11]$.

\subsection{Neurologic Examination}

The Amiel-Tison test was carried out with the examiner blinded to the infant's clinical history. All examinations were performed by a neuropediatrician, with the infant undressed in a warm room when he/she was awake but quiet. The test included several categories, such as cranial assessment, mother's questions, abnormal eye signs, sensory function, posture and spontaneous motor activity, passive and active tone, primitive and tendon reflexes, and postural reactions. Although Amiel-Tison warns that neurologic examination cannot be measured quantitatively, responses were scored on a graded scale $(0,1,2)$ for comparison with other variables. Mild abnormalities included abnormalities in tone and excitability but no central nervous system (CNS) depression and no seizures; moderate abnormalities include abnormal tone with signs of central nervous system depression (poor interaction, hypoactivity, hyporeflexia) and one or two isolated seizures; while severe abnormalities included repeated seizures associated with overt CNS depression ranging from lethargy to coma [6]. 


\subsection{Bayley Scales of Infant Behavior}

The Bayley scales of infant behavior (BSIB) have been widely used to assess mental and motor development in infants [17]. Infants were tested by the different sub-scales such as: cognitive, language, motor, social-emotional, and general-adaptative. The BSIB score was obtained by adding together the number of tests equivalent to the infant's ages; the raw score (RS) was obtained. RS was converted in standardized points, obtaining the index score (IS) with a mean of 100 and standard deviation (SD) of 15. Based in IS, infants were classified within normal $(\geq 85)$, mildly delayed (between 70 and 84 ), and markedly delayed performance $(\leq 69)$. We also carried out a categorical classification for BSIB development as follows: adequate performance is considered when IS $\geq 85$, and inadequate is when IS $<85$. The BSIB was conducted once at 6 months of age in both groups, and the results were statistically compared.

\subsection{Electroencephalography}

Studies were performed in 45 min sessions after-feeding sleep. Infants were studied in supine position. The scalp was prepared with an acetone and alcohol mixture. Electrodes impedances was always $<5$ kilo-ohms. Recordings were obtained on an 8 channel electroencephalographer (Grass, model 78 D, Quincy, MA, USA) and consisted of eight EEG channels. Gold cup electrodes were set in place with electrolytic cream and the infant's head was wrapped with tape strips based on the $10-20$ system modified for newborns. Two bi-polar montages were uti- lized, each montage, one half of the record time. The first was: Fp1-F3, F3-C3, C3-P3, P3-O1, Fp2-F4, F4-C4, C4- P4, P4-O2 and the second was: Fp1-F3, F3-C3, C3-P3, P3-O1, Fp2-F8, F8-T4, T4-T6, T6-O2. Paper- speed recording was $15 \mathrm{~mm} / \mathrm{sec}$, time constant was $0.3 \mathrm{sec}$, sensitivity was $7.5 \mu \mathrm{V} / \mathrm{mm}$, and band pass filters were set between 0.1 and 70 Hertz (Hz). Observations included eyes open, eyes closed, eye movements, body movements, jerks and twitches, startle, sucking movements, vocalizations, and respiratory irregularities such as sighs and pauses. Careful interpretation was performed off-line. Brain waves present in the recording were classified as delta $(0.1-3.75 \mathrm{~Hz})$, theta $(4-7.75 \mathrm{~Hz})$, alpha $(8-12.75 \mathrm{~Hz})$ and beta $(13-35$ $\mathrm{Hz})$ rhythms. Brain activity was visually and topographically mapped on frontal, central, parietal, occipital and temporal areas. Scoring was based on operational definitions using representative neonatal EEG samples of each of the previously mentioned areas [18].

\subsection{Data Analyses}

We performed descriptive analyses to determine arithmetic mean \pm SD for continuous variables and percentages for qualitative variables. Student $t$ tests were conducted to ascertain differences among numerical variables. Categorical results among neonatal EEG, early intervention, and neurologic examination were compared by $X^{2}$ test. The alpha value determined for accepted significance $a$ priori was $\leq 0.05[19]$.

\section{Results}

The age of each infant in both groups were corrected to 42 weeks of conceptional age for any comparison, and the last examination for the objectives of this study was performed at 6 months of corrected age. No differences in age, socioeconomic features, and examination results were observed at the first examination between groups.

\subsection{Neurologic Examination}

All subjects in nEI group had a normal result in the first examination. Five infants in EI-K group (36\%) were suspected of neurologic abnormalities, and nine (64\%) exhibited a normal result at their first appointment prior to EI-K program I initiation. Significantly, major abnormality frequencies were observed in the EI-K group $(p<$ $0.05)$, in which hypotonus and low response to external stimuli were the most frequently observed abnormalities.

Six months later at the second examination, nine $\mathrm{nEI}$ subjects present a normal result, while two had suspicion of neurologic abnormalities; most frequent alteration was delay in neck control. In EI-K group, all infants had a normal result at the second appointment. Significant differences between groups were observed $(p=0.05)$ with better performance in EI-K than $\mathrm{nEI}$ group.

\subsection{EEG Recordings}

At the first evaluation, five subjects in $\mathrm{nEI}$ group had a normal EEG recording, while six infants had a mildly abnormal EEG consisting of maturation delay. Seven infants in EI-K group showed normal EEG result, six had a mildly abnormal result consisting of maturation delay and one infant had a moderately EEG abnormal result consisting of maturation delay and asymmetry. No statistical differences were observed between groups $(p>0.05)$.

Six months later in nEI group eight infants showed normal EEG result, while one was mildly abnormal exhibiting slow background activity. Two parents failed to fulfill the appointment for the second EEG recording. In EI-K group; 12 infants had normal EEG recording, and only two infants had abnormal EEG result showing isolated spikes and slow waves (moderately abnormal result). No statistical difference between groups was observed $(p>0.05)$.

\subsection{Bayley SIB}

Comparison of mental and motor developmental coeffi- 
cients at 6 months of corrected age is shown in Table 2. Data demonstrated significant differences between groups $(p<0.05)$.

\subsection{Qualitative Analysis}

We could not find a correlation between the abnormalities in the neurological examination and in Bayley SIB, due to differences in the types of qualification: amielTison neurological examination is binomial while Bayley SIB provides a quantitative score. On the other hand children with abnormal result in the first assessment improved in the second, while some normal children had abnormal results in the second assessment.

\section{Discussion}

We studied the effects of intensive EI in a short but highly selected sample of premature infants treated early from hospital discharge to 6 months of age. We found differences in neurologic and behavioral examination measurements between nEI and EI-K infants, with a better performance in the EI-K infant group, suggesting a positive effect of EI-K on neurodevelopment when compared with results from the nEI group. However we were unable to observe differences in neurophysiologic and measurements.

One problem associated with the use of the term "early" lies in the interpretation of the term. "Early" can be understood in several ways, for example: 1) early after birth; 2) early in the first year of life; and 3) early after onset of the condition. Each intervention type is associated with advantages and disadvantages. Some studies of EI have been carried out in the neonatal intensive care unit, others begin after hospital discharge, and still others initiate during the second semester of life. In our case, we began EI after hospital discharge when the brain is considered very plastic. The majority of studies on the effects of EI on neurodevelopment are heterogeneous, with large variations in number of participants, intervention methods, age at study initiation, and measurements for evaluating intervention. These methodologic points render comparisons difficult among differ-

Table 2. Bayley's developmental coefficients in the group of infants in the early intervention ad modum Katona (EI-K) program and in infants without the early intervention (n-EI) program in the first 6 months of life.

\begin{tabular}{ccc}
\hline Group & Mental coefficient & Motor coefficient \\
& $x \pm(\mathrm{SD})$ & $x \pm(\mathrm{SD})$ \\
\hline EI-K group & $112.8(9.1)$ & $124(7.7)$ \\
nEI group & $101.8(7.2)$ & $110(8.9)$ \\
$p$ & $<0.05$ & $<0.05$ \\
\hline
\end{tabular}

$x=$ mean; $\mathrm{SD}=$ Standard deviation. ent studies. Thus, we limited our discussion in studies than began EI after hospital discharge and continued during the first year of life.

Several studies on EI have been carried out in high-risk infant populations after hospital discharge [20-28]. When the effects of EI programs on neurodevelopmental treatment were evaluated, the majority of studies did not showed exhibits significant effects of the intervention on motor development [20-24], however, the majority of effects evaluated in the previously mentioned studies were based solely on global developmental tests. Another two studied applied programs to stimulate infant motor development and, consisted of general sensory and motor stimulation. In another study, the authors included enhancement of parent-infant interaction, while yet another was concerned with other passive handling techniques; both of these studies reported significant positive effects of intervention on motor development $[25,26]$ which is in agreement with our results. Other studies evaluated neurodevelopment in small groups of infants treated ad modum Vojta, reporting a dose-response effect of treatment $[27,28]$.

Differences between these studies not finding clear evidence of the positive effects of EI and our data can be attributed to that the Katona method is the most intensive EI program (at least $6 \mathrm{~h}$ a day of work), with special relevance concerning the mother-infant relationship. Although our follow-up time was short, our results hold the promise of good outcome in the clinical neurodevelopment and brain plasticity of premature infants and deserve additional attention in future works. However, the effects of EI-K on the neurophysiologic area are deserving of even more investigation.

Few studies have measured neurobehavioral and EEG parameters in parallel in infants under EI treatment. Two studies in the medical literature report increased coherence in fast-alpha and -beta frequencies between frontal and occipital-parietal areas [29,30] based on brain plasticity. Nonetheless, these investigators used quantitative EEG to measure EI effects on brain electrical activity, while we only utilized the qualitative visually-qualified EEG. This fact may be the explanation of why we were unable to observe subtle EEG differences when comparing both groups in visual analyses. In future papers, we will use quantitative EEG to perform these comparisons.

BSIB was performed in infants of both groups to determine whether there are motor and performance developmental differences between nEI and EI-K groups. BSIB is a well-standardized test and has been employed as a gold-standard to test the sensitivity and specificity of other behavioral tests in premature infants [31]. We observed differences in developmental items between groups, suggesting a great sensitive of this test to observe 
cognitive developmental differences when comparing infants under K-EI treatment with those employing other EI techniques.

In summary, comparison between the nEI and EI-K techniques for treatment in premature infants results in certain advantages of EI-K use at 6 months of corrected age, mainly in neurologic- and behavioral-development items. The main limitations of our study include the small number of patients studied and the short duration of follow-up; thus, our results must be considered as tendencies, and not strong conclusions. Future studies regarding effects of EI ad modum Katona must include a greater number of patients with a longer follow-up period, in addition to other computer- assisted neurophysiologic and biochemical measurements in order to respond questions left open by this study.

\section{Aknowledgments}

In memorian to Helda Benavides-González B. Sc. pioneer of early intervention studies in Mexico. Thanks to Maggie Brunner M. A., for her help in the preparation of the English version of the article.

\section{REFERENCES}

[1] C. F. Martínez-Cruz, A. Poblano, L. A. Fernández-Carrocera, R. Jiménez-Quiróz and N. Tuyú-Torres, "Association between Intelligence Quotient Scores and Extremelly Low-Birth Weight in School-Age Children," Archives of Medical Research, Vol. 37, No. 5, 2006, pp. 639-645. doi:10.1016/j.arcmed.2005.12.001

[2] T. Kanda, M. Yuge, Y. Yamori, J. Suzuki and H. Fukase, "Early Physiotherapy in the Treatment of Spastic Diplegia," Developmental Medicine and Child Neurology, Vol. 26, No. 4, 1984, pp. 438-444. doi:10.1111/j.1469-8749.1984.tb04468.x

[3] A. Poblano, "Early Identification and Treatment of Infants with Neurologic Damage (in Spanish)," Editors de Textos Mexicanos, México City, 2003.

[4] C. Bauw-Hospers and M. Hadders-Algra, "A Systematic Review of the Effects of Early Intervention on Motor Development," Developmental Medicine and Child Neurology, Vol. 47, No. 6, 2005, pp. 421-432. doi:10.1017/S0012162205000824

[5] H. F. R. Prechtl, "The Neurological Examination of the Full Term Newborn Infant," Mac Keith Press, London, 1977.

[6] C. Amiel-Tison, "Update of the Amiel-Tison Neurologic Assessment for the Term Neonate or at 40 Weeks Corrected Age," Pediatric Neurology, Vol. 27, No. 3, 2002, pp. 196-212. doi:10.1016/S0887-8994(02)00436-8

[7] C. L. Pereira, A. M. Aguilar, F. Rivera, L. Cruz, I. Santos and A. Poblano, "Concordance between Neurologic Screening Test and Neurologic Examination in Newborns," Clinical Pediatrics, Vol. 45, No. 4, 2006, pp. 315-323. doi:10.1177/000992280604500403
[8] M. C. Toet, P. M. A. Lemmers, L. J. van Schelven and F. van Belt, "Cerebral Oxygenation and Electric Activity after Birth Asphyxia: Their Relation to Outcome," Pediatrics, Vol. 117, No. 2, 2006, pp. 333-339. doi:10.1542/peds.2005-0987

[9] A. Poblano and R. Gutiérrez, "Correlation between the Neonatal EEG and the Neurological Examination in the First Year of Life in Infants with Bacterial Meningitis," Arquivos de Neuro-Psiquiatria, Vol. 65, No. 3, 2007, pp. 576-580. doi:10.1590/S0004-282X2007000400005

[10] F. Katona, "Developmental Clinical Neurology and Neurohabilitation in the Secondary Prevention of the Pre- and Perinatal Injuries of the Brain. In: P. M. Vietze and H. G. Vaughan, Eds., Early Identification of Infants with Developmental Disabilities, Grune and Strattonm, Philadelphia, 1988, pp. 121-144.

[11] F. Katona, "Clinical Neurodevelopment Diagnosis and Treatment,” In: P. R. Zelazo and R. G. Barr, Eds., Challenges to Developmental Paradigms: Implications for Theory and Treatment, Lawrence Erlbaum, Hillsdale, 1989, pp. 167-187.

[12] H. Capurro, S. Konichezky, D. Fonseca and R. CaldeyroBarcia, "A Simplified Method for Diagnosis of Gestational Age in the Newborn Infant," The Journal of Pediatrics, Vol. 93, No. 1, 1978, pp. 120-122. doi:10.1016/S0022-3476(78)80621-0

[13] R. Ramos-Galván, "Pediatric Somatometry. Follow-up Study in Infants and Children from México City," Archives of Medical Research, Vol. 6, Supplement 1, 1975, pp. 83-396.

[14] G. M. Mueller, C. P. Weiner and J. Yankowitz, "ThreeDimensional Ultrasound in the Evaluation of Fetal Head and Spine Anomalies," Obstetrics and Gynecology, Vol. 88, No. 3, 1996, pp. 372-378. doi:10.1016/0029-7844(96)00207-4

[15] S. A. Leib, D. G. Benfield and J. Guidubaldi, "Effects of Early Intervention and Stimulation on the Preterm Infant," Pediatrics, Vol. 66, No. 1, 1980, pp. 83-90.

[16] H. Benavides-González, M. A. Rivera-Rueda, M. P. Ibarra-Reyes, M. E. Flores-Tamez, A. Fragoso-Ramírez, N. Morán-Martínez and L. A. Fernández-Carrocera, "Effects of Early Multimodal Stimulation on Premature Newborn Infant," Boletin Medico del Hospital Infantil de Mexico, Vol. 46, No. 12, 1989, pp. 789-795.

[17] N. Bayley, "Bayley Scales," Harcourt Brace, San Antonio, 1993.

[18] S. Garza-Morales and A. Poblano-Luna, "Abnormal Electroencephalograms in Newborns," In: A. Gil-Nagel, J. Parra, J. Iriarte and A. M. Kanner, Eds., Textbook of Electroencephalography, McGraw Hill-Interamericana, Madrid, 2002, pp. 117-130.

[19] J. E. Hair, R. E. Anderson, R. L. Tatham, et al., "MultiVariate Data Analysis,” Prentice Hall, Madrid, 1999.

[20] S. Imamura, K. Sakuma and T. Takahashi, "Follow-up Study of Children with Cerebral Coordination Disturbance (CCD, Vojta)," Brain and Development, Vol. 5, No. 3, 1983, pp. 311-314. 
[21] M. Goodman, A. D. Rothberg, J. E. Houston-McMillan, P. A. Cooper, J. D. Cartwright and M. A. van der Velde, "Effect of Early Neurodevelopmental Therapy in Normal and at-Risk Survivors of Neonatal Intensive Care," The Lancet, Vol. 326, No. 8468, 1985, pp. 1327-1330. doi:10.1016/S0140-6736(85)92626-1

[22] M. C. Piper, V. I. Kunos, D. M. Willis, B. L. Mazer, M. Ramsay and K. M. Silver, "Early Physical Therapy Effects on the High-Risk Infant: A Randomized Controlled Trial," Pediatrics, Vol. 78, No. 2, 1986, pp. 216-224.

[23] A. D. Rothberg, M. Goodman, L. A. Jacklin and P. A. Cooper, "Six-Year Follow-up of Early Physiotherapy Intervention in Very Low Birth Weight Infants," Pediatrics, Vol. 88, No. 3, 1991, pp. 547-552.

[24] A. M. Weindling, P. Hallam, J. Gregg, H. Kenka, L. Rosenbloom and J. L. Hutton, "A Randomized Controlled Trial of Early Physiotherapy for High-Risk Infants," Acta Paediatrica, Vol. 85, No. 9, 1996, pp. 1107-1111. doi:10.1111/j.1651-2227.1996.tb14226.x

[25] M. E. Barrera, C. E. Cunningham and P. L. Rosenbaum, "Low Birth Weight and Home Intervention Strategies: Preterm Infants," Journal of Developmental and Behavioral Pediatrics, Vol. 7, No. 6, 1986, pp. 361-366. doi:10.1097/00004703-198612000-00006

[26] R. Lekskulchai and J. Cole, "Effect of a Developmental Program on Motor Performance in infants Born Preterm," The Australian Journal of Physiotherapy, Vol. 47, No. 3, 2001, pp. 169-176.

[27] M. d'Avignon, L. Norén and T. Årman, "Early Physio- therapy ad Modum Vojta or Bobath in Infants with Suspected Neuromotor Disturbance," Neuropediatrics, Vol. 12, No. 3, 1981, pp. 232-241. doi:10.1055/s-2008-1059654

[28] S. Brandt, H. V. Lonstrup, T. Marner, K. J. Rump, P. Selmar, L. K. Schack, M. d'Avignon, L. Norén and T. Årman, "Prevention of Cerebral Palsy in Motor Risk Infants by Treatment ad Modum Vojta," Acta Paediatrica, Scand, Vol. 69, No. 3, 1980, pp. 283-286. doi:10.1111/j.1651-2227.1980.tb07079.x

[29] B. M. Beulher, H. Als, F. H. Duffy, G. B. McAnulty and J. Liederman, "Efectiveness of Individualized Developmental Care for Low-Risk Preterm Infants: Behavioral and Electrophysiological Evidence," Pediatrics, Vol. 96, No. 5, 1995, pp. 923-932.

[30] H. Als, F. H. Duffy, G. B. McAnulty, M. J. Rivkin, S. Vajapeyam, R. V. Mulkern, S. K. Warfield, P. S. Huppi, S. C. Butler, N. Conneman, C. Fischer and E. C. Eichenwald, "Early Experience Alters Brian Function and Structure," Pediatrics, Vol. 113, No. 4, 2004, pp. 846-847. doi:10.1542/peds.113.4.846

[31] M. J. Vincer, H. Cake, M. Graven, L. Dodds, S. McHugh and T. Fraboni, "A Population-Based Study to Determine the Performance of the Cognitive Adaptive Test/Clinical Linguistic and Auditory Milestone Scale to Predict the Mental Developmental Index at 18 Months on the Bayley Scales of Infants Development-II in Very Preterm Infants," Pediatrics, Vol. 116, No. 6, 2005, pp. e864-e867. doi:10.1542/peds.2005-0447 